\title{
Osteopathie international
}

Nach der Annual Convocation der American Academy of Osteopathy in Birmingham, Alabama, im März 2006 ist ein Blick auf die internationale Osteopathieszene recht aufschlussreich. Die „World Osteopathic Health Organisation, WOHO“ hat dort ihren Vorstand gewählt (deutsche Vertreterin in diesem Gremium ist Marina Fuhrmann, D.O. M.R.O., Vorsitzende des VOD). Zwei der WOHO-Vorstandsmitglieder, nämlich Simon Fielding aus England und Prof. Clive Standen aus Neuseeland, sind darüber hinaus auch aktiv im Vorstand der „Osteopathic International Alliance, OIA“. Es ist als ein besonderes Zeichen zu werten, dass man für den Integrationsprozess auf erfahrene Osteopathen, die keine Ärzte sind, sehr großen Wert legt. So wird an höchster Stelle Transparenz praktiziert und augenscheinlich haben die dort Aktiven, die alle ehrenamtlich arbeiten, erkannt, dass diese Einstellung viel Zeit und Geld spart. (Zudem lässt sich so hoffentlich verhindern, dass die beiden Organisationen, die sich laut Satzung für die Verbreitung der Osteopathie weltweit einsetzen wollen, in gegenseitige Konkurrenz treten.) Während die WOHO eine Organisation von Einzelmitgliedern darstellt, Osteopathen wie auch osteopathische Ärzte, ist die OIA eine Organisation von Organisationen (wie Osteopathische Colleges, nationalen Organisationen wie die American Osteopathic Organisation AOA und Regulierungsbehörden wie der General Osteopathic Council in England). WOHO und OIA haben in einer gemeinsamen Erklärung ihre unterschiedlichen Kompetenzen wie auch die Absicht einer reibungslosen Zusammenarbeit bekundet. Das ist umso erfreulicher, als die Gründung der OIA ursprünglich nicht frei war von dem Gedanken eine Konkurrenz zur WOHO aufzubauen. Übrigens: Jede(r) Osteopath(in) kann Mitglied in der WOHO werden. Schauen Sie doch einmal auf die Webseite www.woho.org!

Anlässlich des diesjährigen VOD-Kongresses wird die OIA eine Parallelveranstaltung in Schlangenbad abhalten mit Jahreshauptversammlung und dreitägigem politischen und akademischen Programm. Die Komitees der WOHO werden ebenfalls tagen. Die amtierenden Präsidenten der American Osteopathic Organisation, AOA, John A. Strosnider, D.O., wie auch der AAO, Kenneth Johnston, D.O., FAAO, haben ihr Kommen angekündigt. An ausländischer Prominenz wird in Schlangenbad also kein Mangel herrschen. Das zeigt, dass dem VOD-Kongress auch international eine hervorragende Stellung eingeräumt wird. Der Veranstalter trägt dem beispielsweise Rechnung, indem die Präsentation der Thesen wie auch die Reden des Festbanketts erstmalig simultan übersetzt werden.

Weltweit scheint sich glücklicherweise immer mehr die Erkenntnis durchzusetzen, dass Osteopathie durchaus von osteopathischen Ärzten wie auch von nichtärztlichen Osteopathen ausgeübt werden kann. So schrieb die Vizepräsidentin der WOHO, Jane Carreiro, D.O., in einem Interview der französischen Zeitung „ApoStill“: „Ich sehe keinen Konflikt zwischen der Behandlung durch Osteopathen und der Behandlung durch osteopathische Ärzte. Beide Gruppen haben bei der Behandlung ihrer Patienten unterschiedliche aber parallele Zugänge." Es wird Zeit, dass sich eine derartige Einstellung auch hierzulande in gewissen Kreisen durchsetzt! 